\title{
LIBROS «DE MANO»Y LIBROS «D'EMPRENTA» PARA LA ENSEÑANZA DE LOS SABERES EN EL TRÁNSITO AL RENACIMIENTO*
}

\author{
Manuel José Pedraza Gracia**
}

Universidad de Zaragoza

\begin{abstract}
Resumen: Se analiza la presencia de productos y libros impresos o manuscritos en los distintos niveles de aprendizaje durante el fin de la Edad Media y las dos primeras décadas del siglo XVI indagando en los documentos notariales. Especial mención merecen el aprendizaje de las primeras letras y las enseñanzas universitarias como límites extremas en la formación.

Palabras clave: Enseñanza; primeras letras; enseñanza universitaria; libros impresos; libros manuscritos.
\end{abstract}

Title: MANUSCRIPTS AND PRINTED BOOKS FOR TEACHING IN THE TRANSITION TO RENAISSANCE. Abstract: The article analyzes the presence of printed books and manuscripts at different levels of learning in the late Middle Ages and the first two decades of the sixteenth century inquiring into the legal documents. Special mention deserve the learning of the first letters and university education as extreme limits in training.

Keywords: Education; first letter; university education; printed books; manuscripts.

\section{INTRODUCCIÓN.}

Según se desprende de los principales estudios (McLuhan, 1969; Eisenstein, 1979; 1994; Ruiz García, 2011) sobre la influencia de la imprenta en la cultura, se mantiene que «a finales del siglo XV, la reproducción de materiales escritos empezó a desplazarse desde el pupitre del copista al taller del impresor. Este cambio... revolucionó todas las formas de conocimiento...» (Eisenstein, 1994, p. 15). Se sobrentiende que también el texto impreso, desde el mismo inicio de las actividades de la imprenta, comienza a tener un peso importante en el ámbito de la transmisión del conocimiento por antonomasia, la enseñanza de los saberes, como parte básica y, también, esencial del conocimiento humano; $y$, como consecuencia en el del aprendizaje.

El interés de los poderosos por establecer una normalización de los textos que no era del todo posible con la copia manuscrita era perfectamente compatible con el hecho de que el mismo (idéntico) texto pudiese llegar a un número mucho mayor de lectores, y con mayor celeridad, porque a la par se producía un incremento notabilísimo del número de unidades documentales. De esta manera se entiende que la Iglesia sea uno de los principales artífices de la introducción de la imprenta en algunas capitales hispánicas y, aún después, en América; y que el Estado (mejor los estados) se apunten con rapidez al aprovechamiento que la nueva técnica ofrece para la divulgación de los méritos y bondades de quienes ejercen la autoridad. Los poderes, en consecuencia, se inclinaron en el apoyo de la imprenta en su proceso de normalización de textos de carácter jurídico-procedimental o histórico-propagandístico. En efecto, el impreso, por su confección mecánica aportaba como producto acabado ejemplares idénticos, o esencialmente idénticos, lo que proporcionaba una seguridad (más aparente que real) de que los contenidos del libro se ajustaban de forma fiable a lo que debía figurar en él; por consiguiente, el hecho de que la imprenta proporcione fiabilidad en los textos debido al principio de repetibilidad será un factor primordial en el éxito de la difusión de la imprenta (Einsenstein, 1994, p. 35). También se produce un fenómeno que se debe de considerar: el análisis de un ejemplar era suficiente para entender que el resto de los ejemplares de la edición se encontraban en el mismo estado y que sus contenidos eran idénticos; todo ello frente a las posibles diferencias que puedan presentar las distintas copias manuscritas de la misma obra. La imprenta ofrecía una perspectiva mucho mayor de seguridad. El (libro) impreso desde momentos muy tempranos de su evolución incluía con frecuencia los datos de la fabricación, que con cierta celeridad se hicieron obligatorios, por lo que se podía localizar con cierta facilidad al responsable material de su elaboración. Pero, sin duda, no fueron estas ventajas las que influyeron en el éxito del impreso, sino el hecho de que los costes de producción se veían claramente reducidos.

Parece evidente que el impreso ofrecía algunas importantes desventajas sobre el manuscrito a los ojos de los contemporáneos cultos lectores. Quizás lo que hoy nos pueda parecer un aspecto positivo no fuese entendido de la misma manera: el impreso ofrecía una lectura monótona de letras exactamente iguales, artificiales, menos vivas; quizás el libro impreso carecía de las rúbricas en diversos colores que individualizaban con precisión los apartados de

\footnotetext{
* Trabajo realizado con el apoyo del Proyecto de la Secretaría de Estado de Investigación del Ministerio de Ciencia e Innovación HAR 2011-23196.

*** pedraza@unizar.es
}

Recibido: 03/04/2013; 2 ${ }^{\mathrm{a}}$ versión: 23/05/2013; aceptado: 23/05/2013.

PEDRAZA GRACIA, M.J. Libros «de mano» y libros «d'emprenta» para la enseñanza de los saberes en el tránsito al Renacimiento. Anales de Documentación, 2013, vol. 16, nº 2. Disponible en: http://dx.doi.org/10.6018/analesdoc.16.2.172341 
los diferentes libros en los que se dividían las obras; quizás el nuevo libro no poseía con la misma frecuencia la belleza de las ilustraciones del libro manuscrito que se van viendo sustituidas por unas imágenes más impersonales proporcionadas por tacos xilográficos que no aportan, salvo en las raras ocasiones de la aplicación manual de pigmentos acuosos, más color que el negro de la línea del dibujo sobre el blanco de la página. «Así pues, después de mediados del siglo XV, la escritura manual de puño y letra representó aun todavía más intimidad y deferencia, puesto que existía una nueva forma escrita, la de molde, que era ya absolutamente impersonal» (Bouza, 1992, p. 37). Las palabras de Gonzalo García de Santa María en su testamento son un reflejo de esta percepción, aunque el principal lamento del humanista zaragozano no se centre en el menoscabo que se ha producido en el atractivo estético de los libros, sino en lo que respecta a la depreciación de su valor económico en general:

Item, lexo a mi mujer todo lo que se fallara dentro de mi studio, assi de libros que esten en las tablas y fastigores, como en el suelo y dentro de caxas y caxones; assi de pergamino como de paper, y assi scriptos de mano, como de emprenta, y assi cisternados en pergamino como otros qualesquiere, y assi griegos como latinos, y assi de romance como de latin, de qualquiere facultad sean; que creo valen hoi, ahun con la emprenta, mas de cinquo mil sueldos, y valian mas de mil florines de oro antes de la emprenta... que segun son muchos y de diversas facultades, si se vendian juntos creo que ahun valdrian hoi mas de V mil sueldos, porque hai libros peregrinos con los quales, dando en buenas manos, se podria fazer uno mucha honra, porque tengo los mas de ellos muy studiados y corregidos... Y no se maraville alguno que tanta diligencia pongo en mis libros, porque segun mi affection, mas valen que todo el resto de mi mueble ${ }^{l}$.

Es notable la reflexión de García de Santa María sobre la «honra» que podría hacer su colección bibliográfica si cayese en buenas manos dotando al libro de un valor de conocimiento, de transmisor de saberes, al que no duda en adjudicar un valor de gran significación, ya que es precisamente su biblioteca lo que más vale entre sus bienes muebles. Otras apreciaciones del mismo tipo y en sentido contrario se encuentran en boca de libreros y otros profesionales del libro (Einsenstein, 1994, p. 30). Pero no sólo afectaba a los aspectos formales de los textos sino que también se producía «una gran desconfianza ante la imprenta, pues se estimaba que quebraba la familiaridad entre el autor y sus lectores y corrompía la corrección de los textos al entregarlos a manos "mecánicas" y a las prácticas del comercio» (Chartier, 2000, p. 14). De hecho el fenómeno que podía producirse mediante la introducción de errores inadvertidos era precisamente el de la difusión de los mismos, ya que cualquier errata se vería reflejada en todos y cada uno de los ejemplares de la edición que habrían de servir, sin la menor duda, para la confección de nuevos impresos que, a su vez, podrían incurrir en nuevos errores en todos sus ejemplares y, así, sucesivamente, resultando la multiplicación de ejemplares la causa de la multiplicación de errores (Eisenstein, 1994, p. 79; Grafton, 2011).

\section{LAS PRIMERAS LETRAS.}

El impreso que servía, como el manuscrito, para el fin de la enseñanza debió de verse también incluido en este conjunto de obras que estaban más predispuestas que otras para pasar a las prensas y los tipos movibles. Los libros de enseñanza son de uso muy común y, por tanto, ayer, como hoy, un negocio muy rentable. Además, la normalización de los mismos supone la potenciación del aprendizaje conjunto frente al individualizado. Todo ello sin entrar en el hecho de que el hábito de una lectura tipográfica sería una excelente punta de lanza hacia la preferencia por parte de los lectores de los productos tipográficos frente a los manuscritos.

Varias son las maneras en las que el individuo alcanza los saberes de la lectura y la escritura. En los momentos inmediatamente posteriores a los que hace referencia este trabajo se han llegado a cuantificar de la siguiente manera: aproximadamente dos tercios de la población lectora llegó a ese conocimiento en una escuela con un maestro, un catorce por ciento reconoce haber aprendido con el cura o el sacristán y el dieciséis por ciento dirigido por un familiar (Viñao, 1999, p. 53).

Cuando la enseñanza de las primeras letras se debía de realizar en casa del maestro, los servicios de éste se pactaban mediante contratos de aprendizaje de forma individual entre maestro y alumno. Como han puesto ya de manifiesto otros autores (Sánchez Herrero y Pérez González, 1998, p. 49; Álvarez Márquez, 1995; entre otros), los maestros no son profesionales dedicados a otros menesteres que de forma esporádica se dedican a la enseñanza de la lectura y escritura, sino profesionales que tienen como actividad principal la enseñanza de las primeras letras ${ }^{2}$. Es posible y, con seguridad, así se hacía que el maestro concertara varios contratos de forma individual que ejecutaba sucesiva o simultáneamente. Pero este tipo de contratación de aprendizaje no difiere en casi nada con los que se realizan para el aprendizaje de un oficio o profesión, que poseían un componente eminentemente práctico. No parece tampoco probable que todos los alumnos del maestro se encontrasen en el mismo nivel de aprendizaje lo que sin duda exigiría una atención más personalizada. 
Otros estudiantes, por el contrario, sí aprenderían de forma personalizada. Sería ese algo menos de un tercio que llegaba a este conocimiento guiado por los religiosos y los familiares.

Se perseguía esencialmente dotar a los estudiantes de los rudimentos imprescindibles para la lectura y escritura. Como ha puesto de manifiesto Bouza (1992, p. 52) lo primero que se aprendía era a pronunciar que venía a ser lo mismo que leer, ya que la lectura solamente era concebida como lectura en voz alta y, posteriormente, a escribir.

Pero, qué influencia tiene el impreso en estas enseñanzas. La enseñanza más elemental en este momento contaba con algunas herramientas: cartillas, doctrinas y catecismos (Infantes, 1998, p. 35-45). Para la enseñanza de las letras serían las cartillas los instrumentos más utilizados, aunque en las enseñanzas individualizadas sería factible que se emplearan otros instrumentos más acordes a una instrucción de tipo religioso (la lectura y silabeo de las oraciones, por ejemplo), que siempre planeó sobre estas primeras fases de la formación (Infantes, 1998, p. 26).

Las cartillas eran el resultado de la impresión de un único pliego de papel doblado tres veces dando lugar a una cuaderno en octavo de ocho hojas o dieciséis páginas como ha reflejado Viñao (1999, p. 64), si bien las que se han conservando poseen preferentemente el formato de cuarto (Infantes, 1998) dando lugar a cuatro hojas y ocho páginas. Por lo que se conoce de los usos en la imprenta su producción era bastante rápida, unos 1.500 ejemplares en una jornada de trabajo de una única prensa con dos operarios. Debido a que se trataba de un producto que no sufría modificaciones compuesto con tipos de gran cuerpo, tras la limpieza correspondiente, las formas debían de quedar montadas para hacer sucesivas tiradas cuando fuese necesario. Naturalmente, la existencia de la herramienta impresa presupone su propia necesidad y su utilidad puesto que no se invierte dinero y esfuerzo en producirlas si no existe la posibilidad de obtener un beneficio por ello. La importancia económica que alcanzó andando el tiempo el negocio de la producción de cartillas llevó a conceder privilegios de impresión de estos instrumentos para la enseñanza de la lectura, pero, para ello, hay que llegar a la segunda mitad del siglo XVI.

Si se establece como punto de partida la enseñanza de las letras, los beceroles impresos aparecen desde fines del siglo XV entre los inventarios de libros siendo las primeras menciones a herramientas para el aprendizaje de la lectura impresas que se conservan en la Península (Serrano y Morales, 1898-1899, p. 491; Berger, 1987, p. 2 y 425; Socias, 1992). Estos beceroles, de los que también se han conservado precedentes manuscritos, eran, en esencia, reproducciones del alfabeto y de algunas sílabas (Socias, 2006, p. 18) que permitían un conocimiento muy incipiente de las letras y de la formación de sílabas.

Es seguro que, además de existir estos precedentes manuscritos ya mencionados, debían de existir también carteles de abecedarios que puestos en los lugares de aprendizaje servirían para enseñar las letras a grupos de alumnos o en las manos de los estudiantes para enseñar de forma más individualizada. En la cita clásica de Vives (1999) sobre la forma de enseñar las letras se intuye esta costumbre:

MAESTRO.- Toma el abecedario con la mano siniestra y este puntero con la derecha para señalar cada una de las letras; tente derecho, guarda tu sombrero bajo el sobaco. Oye con atención como yo nombrare las letras, y pon cuidado como las pronuncio. Procura decirlas después, cuando yo te lo pida, del mismo modo que yo las digo. Síguerne ahora a mí, que voy delante diciéndolas una a una. ¿Has entendido bien?

LUCIO-Creo que sí.

MAESTRO.- Cada una de éstas se llama letra: de ellas cinco son vocales, $A, E$ I O, U, que están contenidas en el vocablo español oueia, que en latín se llama ovis. Acuérdate de este nombre. Hacen silaba éstas con cualquiera o con más de las otras; sin vocal no se hace sílaba, y aun una vocal sola es sílaba no pocas veces. Todas las demás se llaman consonantes, Porque no suenan si no se les junta vocal; así tienen un sonido imperfecto y manco. $B C, D, G$, que sin la E suenan poco. De las sílabas se forman las voces o palabras, y de éstas nace el hablar de que todas las bestias carecen; y tú no serás diferente de las bestias si no aprendes a hablar bien. Despabilate y pon cuidado. Anda, siéntate con tus condiscípulos y aprende la lección que te he dicho.

LUCIO.- ¿No jugamos hoy?

ESQUINES- No, porque es día de trabajar. ¿O has venido aquí para jugar? Este no es lugar de juego, sino de estudio.

LUCIO.- ¿Por qué le llaman juego?

ESQUINES.- Se le llama juego, pero literario, porque aqui se ha de jugar con letras... 
Vives menciona la existencia de encerados o tablas en las que las letras están dibujadas para que sean identificadas por los alumnos. Nada se sabe sobre la condición de impresos de estas tablas, pero cabe suponer que, aunque sólo sea por la poca venta que poseerían, no se debieron de producir de forma impresa, sino que es más probable que fuesen productos manuscritos. Es evidente que una enseñanza que precisa una herramienta normalizada para cada estudiante, la cartilla, que sugiere especialmente un trabajo personal, es mucho más rentable por lo que a su producción se refiere que aquélla que se emplea para enseñar a muchos de forma comunitaria, la tabla. Estos instrumentos colectivos parecen servir para el estudio memorístico del grupo por más que Vives lo ejemplifique en su diálogo con un único alumno, al que manda, en efecto, sentarse con sus condiscípulos y aprender la lección.

El becerol o la cartilla (impreso o manuscrito) sería la herramienta fundamental en el aprendizaje de ese porcentaje minoritario, pero amplio, que aprendía estos conocimientos de mano de los curas o de sus propios familiares.

Junto a estos instrumentos, tablas y cartillas, son perfectamente conocidos otros tipos que sirvieron para este mismo fin. Se mencionan de forma no del todo infrecuente colecciones de letras en diversos soportes que servirían para conocerlas y que por su movilidad facilitarían el silabeo y la formación de palabras. Precisamente en estos instrumentos de lectura se ha fundamentado el origen de la idea de la imprenta con tipos movibles de madera en torno a la figura de Laurens Janszoon Coster. Aunque no se conocen de forma material muchos ejemplos de estas herramientas han llegado, sin embargo, algunas referencias documentales como la de las «ventidos cartas de aprender letras para ninyos») que figuran en el inventario de los bienes del mercader Gaspar Terrén contratado en $1503^{4}$ y la alusión que a ellas hace Juan de Ycíar (1548) en momentos próximos, aunque posteriores, a los estudiados. Queda encajado el juego literario al que hacía referencia Vives en su diálogo con el juego de cartas cuyo fin era el mismo que Vives refleja en el propio diálogo al hacer referencia a las vocales y consonantes. No se menciona en ese documento si estas cartas estaban impresas o eran manuscritas, pero teniendo presente la vigencia que los naipes, como herramienta de juego, habían alcanzado en las sociedades del tránsito de la Edad Media hacia la modernidad cabe pensar con escasas dudas que se trataba de un juego de cartas impresas como lo eran los propios naipes de juego. Siguiendo este mismo referente, cabe también suponer que se trata de un producto exclusivamente xilográfico como lo era su referente lúdico que se confeccionaba con una o dos tablas xilográficas con las que se estampaba el papel especial para este fin de textura más firme y más grosor, denominado por ello, papel de naipes, cuyo comercio no es infrecuente en la época ${ }^{5}$. Incluso sería bastante factible que fuesen los mismos artesanos que los confeccionaban (naiperos) los que también fabricasen estas otras cartas para el aprendizaje de la lectura.

Por lo que respecta a la escritura, resulta evidente que el proceso de aprendizaje requería pocos materiales salvo los propios de la actividad manuscrita. Cuando aparecen artes de escritura no lo son para el aprendizaje de las primeras letras sino para el conocimiento de los diferentes tipos de letras manuscritas y como muestras para escribanos (Ycíar, 1548). Los manuales de escritura que surgen en el renacimiento no son libros para la enseñanza de la escritura sino para la enseñanza de profesionales escribanos (Martínez Pereira, 2006).

Un segundo tipo de libros en la enseñanza serían los utilizados en el paso inmediatamente siguiente al que no llegarían todos los alumnos que hubiesen aprendido a leer y escribir en el paso anterior, porque el hecho de que el individuo aprenda a leer no quiere decir que sea capaz de aprender leyendo (Einsenstein, 1994, p. 44). El principal de los libros pertenecientes a este grupo es el Catón, La persistencia de los procedimientos manuales hace pensar que se seguirían los métodos y los instrumentos propios del manuscrito, circulando copias manuscritas con cierta profusión, como la localizada en Zaragoza en un inventario de $1510^{6}$. Esta recopilación anónima de máximas alcanzó en el mundo de la imprenta numerosas ediciones desde momentos muy tempranos ya que se conocen alrededor de cien ediciones antes de 1510 entre las que hay de Salamanca, Valencia, Sevilla y Zaragoza. Otros libros para la enseñanza son los doctrinales, entre los que también se localizan copias manuscritas ${ }^{7}$, y otras muchas obras que también llegaron rápidamente a la imprenta: el De regimene principum, l'arbre des batailles o la obra de Paulo Vergerio, (Pedraza, 1993, docs. $43,242,775,777,855)$ entre otras muchas... de las que se encuentran ejemplares de mano y de imprenta.

\section{LA ENSEÑANZA SIN LIBROS, APRENDER LOS OFICIOS CONOCIDOS (...Y CON LIBROS, APRENDER LOS OFICIOS PROHIBIDOS).}

Un segundo nivel es el de la formación para el aprendizaje de un oficio. Hay determinadas profesiones que tienen una relación evidente con el libro; los libreros, los calígrafos y los impresores, entre otros ${ }^{8}$. Las firmas que se realizan son tremendamente similares a las que se han encontrado para aprender a leer y escribir, en esencia: los padres o un familiar del aprendiz contrata la entrega del muchacho con un profesional durante un determinado tiempo con el objeto de que el aprendiz aprenda el oficio. El maestro se obliga a darle de comer y mantenerlo en su casa y 
frecuentemente a vestirlo al final del periodo estipulado; y el aprendiz se obliga a permanecer durante el tiempo mencionado al servicio del maestro, bajo penas de diversa consideración que se ejecutaban con cierta frecuencia ${ }^{9}$.

Este tipo de aprendizajes es esencialmente práctico por lo que el impreso no suele entrar en esta actividad y resulta curioso que la enseñanza de la lectura rara vez se menciona de forma explícita en este tipo de contratos. Se da por sentado que el calígrafo o «scriptor de libros de iglesia» debería haber pasado por una escuela de forma previa o que, en su defecto, deberá adquirir ese conocimiento de nuevo. La edición de muestrarios de letras y manuales de escritura impresos es un fenómeno que se inserta en la transmisión de los saberes en ámbitos posteriores con un componente humanístico más acentuado, que seguramente se superpone a la existencia de libros manuscritos con muestras que el propio calígrafo habría ido conformando durante su formación. Una buena parte de los impresores (los operarios de la prensa) no precisaban saber leer ni escribir, lo que resultaba imprescindible para los cajistas, cuya formación debería exigir como mínimo lectura, escritura en lengua vernácula y en latín y cómputo, además de la habilidad exigible a un cajista de imprenta a la hora de conformar las palabras, líneas y páginas de los textos. El librero, salvo que se tratase del maestro o del comerciante de libros al por mayor, tampoco precisa saber leer, aunque le resulte imprescindible identificar las letras para ordenar los cuadernos a la hora de "ligar los libros". Sólo muy tarde se han localizado algunos contratos en los que el aprendizaje de la lectura y la escritura se añaden al conjunto de conocimientos que debe poseer el librero, pero no de forma sustancial ${ }^{10}$. Hay otras profesiones para las que el libro debería tener interés, aquellas en las que las muestras y patrones son imprescindibles: sastres, pintores, grabadores, escultores. Como ocurre con los escribanos de libros de iglesia estas muestras suelen figurar entre los libros propios de los inventarios de los artistas, pero se trata siempre de libros de mano y sólo muy tarde aparecen libros que contienen, por ejemplo, la forma en la que se pueden elaborar los patrones de sastrería (Ensenstein, 1994, p. 64).

Otros saberes para los que los libros resultaban imprescindibles eran los que se denominan «ocultos». En ese mundo clandestino circulaban libros que permitían conocer los procedimientos para acceder a los poderes de lo oculto, pero en este momento siempre se trató de ejemplares manuscritos que podían circular de esa manera entre los iniciados y que llegan a ser robados para ser copiados ${ }^{11}$. La clandestinidad de estos libros lleva a que perdure su producción personal manuscrita prácticamente hasta el siglo XVIII (Chartier, 2000, p. 153).

\section{LIBROS PARA LA ENSEÑANZA UNIVERSITARIA.}

El impreso alcanza su mayor peso dentro de la enseñanza superior en los estudios generales y universidades. Parece resultar perfectamente claro que las enseñanzas superiores requerían esencialmente libros y el paso del «exemplar» y las «pecia» a los ejemplares impresos procedentes de las prensas internacionales suponía garantías de la pureza de los textos y, por consiguiente, de su autoridad.

El libro está inserto, por definición, en la vida del estudiante sin que se pudiese entender la vida de éste sin libros, para el estudio y el aprendizaje y para la subsistencia, ya que eran objetos que se podían empeñar ${ }^{12}$. Los libros se poseen para el estudio y suponen un coste importante en él. En 1498 el notario Longares escribe un billete en el que se puede leer:

Reverendo domine, enbio a vuestra señoria xii sueldos y ruego bos que bos placia de recebirlos y asi mesmo bos placia de escrebirlos por la memoria asi mesmo bos ruego que me perdoneys que tanto tardo en darbos dineros, pero con la ayuda de Dios, yo bos ne imbiare muy presto y bos contentar e como es encargo, asi mesmo bos ruego que por quaridat que hayais por mucho encomendados a mis fijos en que ellos deprendan bien y que bos plaçia de leyrles el "Terencio" y el "Tulio" y otros libros et liendas que a bos señor bos parecera les probechen que como a fijos vuestros bos los encomiendo y que trebaxen continuamente en deprender y ganar y lenas y su ciencia, quedando muy presto a vuestro mandar et servicio, Joan de Longares, notario, presto a vuestro mandar et serbicio.

Item, paguemos para el present de maestro mayor y para cossas yo y mi hermano 11 sueldos, 6 dineros.

Item, paguemos al maestro mayor yo y mi hermano son a saber $x$ sueldos jaqueses.

Item, paguemos de bagillerado yo y mi hermano son asaber vi sueldos jaqueses.

Item, me compro mi padre señor para mi hun "Tulio" que le costo hun florin.

Item, me compro mi padre señor hun "Terencio" para mi que le costo xii sueldos.

Item, paguemos de cambrerado yo y mi hermano son asaber $V$ sueldos jaqueses, xiiii sueldos, vi.

v sueldos Cambrerado restan de mi et de mi hermano, xxxx, xxvi sueldos, viii. 
$x$ sueldos de dormir en la cambrera, lxxxxvi sueldos ii.

Primo es mas bueno y mas sabio, y, por tanto, la licion leyda del maestro es buena ${ }^{13}$.

Los libros, el Terencio y el Tulio, ya tenían en esta época ediciones de imprenta, pero el precio en el que se tasan hace suponer que debía de tratarse de copias manuscritas. Los libros suponían un gasto considerable hasta el extremo de merecer constar en la contabilidad paternal de los gastos de la enseñanza de los hijos y era tan importante que se llega a recordar en algunos testamentos «...Item, lexo de gracia special al dicho mosen Pedro de Ascara, fijo mio, mil sueldos dineros jaqueses con los quales le ruego se tenga por contento, atendido lo mucho que yo he gastado en el en el estudio y en copiarle libros y en otras muchas cosas...» ${ }^{14}$. Se ha de notar que el gasto era importante porque se trataba de mandar copiar los libros, no de obtener ejemplares impresos.

Pero la preocupación paterna por los estudios de los hijos y por que en ellos dispongan de libros es casi una fijación entre los padres que ya habían estudiado, hoy como ayer. Esto resulta patente en el médico Gaspar Lax, padre del filósofo del mismo nombre que en 1502 dicta testamento:

Item, dexo de gracia especial a Gaspar de Lax, fijo mio, todos los libros de medicina, si medico sera. Et si medico no querra seyer, quiero e mando sean para Cherubin de Lax, fijo mio, al qual en el dicho caso se les dexo. Et si el dicho Cherubin de Lax medico no querra seyer, en tal caso quiero e mando que por la herederaz mia infrascripta sean vendidos al mas dant, et el precio que d'ellos preyra sea para utilidat de todos mis fijos e fijas.

Item, dexo de gracia especial a Cherubin de Lax, fijo mio, todos los libros mios de logica e de gramatica para que estudie. Et si estudiar no querra, que sean vendidos segunt arriba dicho es de los otros libros ${ }^{15}$.

$\mathrm{O}$ en el testamento del maestro en artes y humanidades Juan Monterde un año más tarde:

Item, lexo al dicho mi fixo Jeronimo de gracia special todos los libros mios de medecina y filosofia y de humanidat con las speras y strelabios que a la dicha arte le pueden aprovechar, con condicion que lexe a sus hermanicos dellos y pora su provecho ${ }^{16}$.

Y en otros muchos como el presbítero Juan Fernández Arrojo, entre otros:

Item, dexo de gracia special a Anton Rey, hermano de las dichas Maria, Johana e Katarina Rey, el qual studia para ser de la yglesia los libros siguientes.

Primo, un libro lamado manotectus.

Item, floretus cum suys glosis.

Item, Johanes de Turre Cremata super psalterium cum glosa multoribus doctoribus.

Item, libellus de doctrina virtutum et sua viciorum.

Item, libellus de vicis et virtutibus.

Item, Boecius de consolaciorum.

Item, otro clamado exposicio evangeliorum cum glosa optima.

Item, postilla Nicolay de Lira super salterium una cum canticis.

Item, un libro scripto de mi mano en pergamino con muchas obras en el qual sta la etica y cononica y politica y Paulo de Vergerio, los quales libros le dexo para que studiando y leyendo en aquellos se pueda exercitar en buenos actos y virtuosos ${ }^{17}$.

En todos ellos se incide de manera especial en la importancia que posee el libro en la formación, pero en el último se aprecia que los libros de estudio, a pesar de la aparición de la imprenta siguen siendo libros de mano y en este caso de la mano del propio estudiante.

La conjetura, que sólo puede plantearse como hipótesis, es que el conjunto que podría denominarse libro universitario sigue siendo libro de mano a pesar de que la imprenta lleva ya algunas décadas incorporada en las sociedades que habitan la Península. Un nuevo dato incide en esta misma aseveración al revisar el inventario de Domingo Navarro, maestro en artes del Estudio General de Zaragoza.

Item, primerament se fallaron en una caxa que se cierra de colpe sesenta y quatro piecas de libros, los quales eran de letra de mano, y sinse intitulatas y medios rasgados, los quales son de gramatica, logica y filosofia. 
Item, otra arca con cerradura por de fuera donde se fallaron dizisiete piecas de libros, todos de mano y rasguados, de gramatica, logica y filosofia.

Item, otra arca con carradura (sic.) de buelta con XXII piecas de libros, rasguados y de mano, de gramatica y filosofia.

Item, otra arca bieja con XXIIII piecas de libros ut supra.

Item, otra arca con trenta y quatro piecas de libros ut supra.

Item, otra arca con su panyal en la qual arca se fallaron los siguientes libros.

Primo, el texto de la Biblia.

Item, el Obispo de Balencia.

Item, otro llamado Guarinus Verunensis bocabularius.

Item, el Scoto.

Item, el manipulus curatorum.

Item, la glosa del Nicolau sobre el salterio.

Item, otro salterio de mano.

Item, que otro llamado questiones Buridan.

Item, otro llamado sposicio misse.

Item, otro llamado epistolas d'Eusevi.

Item, questiones colibeti.

Item, Tomas d'Aquino super methaphisicam.

Item, una Nebrisa de comento.

Item, questiones super tota philosofia.

Item, Franciscus Mayrones.

Item, otro llamado Ponponi epistola ad Agustinum.

Item, otro llamado Marci Tuli Ciceronis de finibus bonorum et malorum.

Item, otro llamado logica frater Guillermi de Ocan.

Item, comentum Juanis Buridan.

Item, otro llamado Guido.

Item, llamado Petrus Ispanus.

Item, otro llamado disticos y oraciones.

Item, otro llamado Terencio.

Item, exposicio Georgi.

Item, un Boecio de mano.

Item, unos ignos.

Item, un Boecio d'enprenta desligado.

Item, un Boecio de mano.

Item, unas entroduciones de logica.

Item, las questiones de George.

Item, un Boecio de consolacione.

Item, un Guido. 
Estos son los libros que se a fallado en casa, en la primera cambra, en casa de Gracia Romeu.

Item, primerament se fallo un libro llamado comento sobre la politica de mano.

Item, otro llamado el comento del Gaytano super libros deanima.

Item, otro llamado Salustio.

Item, otro llamado ebangelistero con oraciones y omelias de mano.

Item, la primera part de Santo Tomas de mano.

Item, el Obidio de faustis cum comentis.

Item, la sposicion de Jorge super arminias.

Item, un luminario demprenta.

Item, unas questiones de mano sobre los fisicos y de anima.

Item, el primer libro de las sentencias d'enprenta de misterio trinitatis.

Item, unas questiones de mano del Buridan.

Item, la phisica de Ocan de mano.

Item, el primer libro de los de misterio trinitatis.

Item, el quarto de Santo Tomas de mano en pargamino.

Item, el Dicipulo.

Item, la sposicion de Jorge super ocho libros de las phisicas.

Item, el Boecio de consolacione.

Item, el comento de Santho Tomas de Aquino super libros politicorum.

Item, se fallaron trenta y una piecas de libros de mano y de emprenta entre los quales abia de gramatica, logica y philosofia, y todos rasgados y de mala manera.

Item, unos disticos y oraciones a una.

Item, un Terencio de comento.

Item, el Jorge con el testo del Britocus.

Item, los Ebangelios sinse comento.

Es decir, el inventario del profesor del Estudio incorpora 246 libros mencionados de forma específica o general. De ellos 164 de los libros mencionados son aún de mano, frente a 3 cuya condición de imprenta se indica con claridad, en 44 no se menciona este dato y se cita un grupo de 30 en los que se encontrarían mezclados los libros de mano y de imprenta. Esto es, en un cálculo aproximado podría decirse que el conjunto de libros impresos sólo podría llegar a suponer un veinte por ciento del total de los libros de Domingo Navarro. De nuevo, si esta es la colección bibliográfica de un maestro del estudio, parece evidente que el libro de mano tiene una preponderancia enorme frente al libro de imprenta. Además, este libro de mano no siempre se identifica plenamente con obras específicas, sino que en muchas ocasiones son conjuntos de textos de diversos autores que conforman a gusto del poseedor pequeñas recopilaciones sobre temas concretos, generalmente de mano del propio propietario (Chartier, 2000, p. 143). Seguramente, la dificultad de obtención de determinados libros parece ser la causa de la preferencia de libros manuscritos sobre los libros impresos. Tampoco es infrecuente que algunas copias manuscritas tengan como original un ejemplar impreso, es más, algunos autores afirman que

...gran número de los manuscritos hechos a finales del siglo XV fueron copia de los primeros libros impresos. Así el trabajo manual y el impreso siguieron pareciendo casi indistinguibles, incluso después de que el impresor empezara a separarse de las convenciones de los amanuenses y a explotar algunas nuevas características inherentes a su arte. (Einsenstein, 1994, p. 32).

Esta abundancia de libros confeccionados a la medida de cada cual favorecía la presencia de estudiantes que se dedicaban a la encuadernación de los libros que confeccionaban los profesores y los alumnos del estudio. Es el caso del estudiante Juan Climent cuyo inventario, no deja lugar a dudas, es el inventario de un encuadernador:

...Item, XXXXV pieças de ferramientas de fazer libros. 
Item, unas oretas de paper con sus cubiertas de fusta.

Item, hun libro de sermones de emprempta.

Item, hun libro de ignos y floret.

Item, el canto y homelias todo en hun libro.

Item, unas cartas de mensajes.

Item, unas taulas de Lorenço de Valla.

Item, hun libro de emprempta clamado reportorio de los

tiempos.

Item, tres prensas de fusta.

...Item, XXI taulas para cubrir libros.

...Item, una prensa de fusta... ${ }^{18}$.

Es de remarcar que para los libros que no son de imprenta no se indica esta característica. Tampoco queda perfectamente claro si los libros mencionados pertenecían realmente al finado o eran los que tenía en su poder para proceder a su encuadernación.

Si se busca entre los libros de los estudiantes, resulta interesante apreciar como en la mayor parte de los intercambios de libros entre particulares suelen encontrarse ellos o con su intervención. Así, en el inventario de los bienes muebles que vende el estudiante Francisco Bur en 1501 al también estudiante Iñigo Muñoz figuran cuatro libros, todos de imprenta, que se citan de la siguiente manera: «... Quatro libros d'emprenta: los dos libricos de comento, el otro Terencio, el hotro huno de canto...» ${ }^{19}$; una nueva venta del mismo tipo con dos libros se produce entre los estudiantes Domingo Sanz y Colau de Fraylla en $1514^{20}$ figurando un «Terencio» y un «Nebrixa»; y otra más de cinco libros con algunas repeticiones en 1517 entre el estudiante Martín del Puente y Martín de Ejea ${ }^{21}$ : «... E cinco libros, los quales son: un Terencio, unas Epistolas de San Pablo, unos difuntos, unos ignos e un arte de Anthonio,...». Aunque no se mencionen las características físicas de los libros citados, es curioso que cuando se hace, todos los libros sean de imprenta. El análisis de los marginalia de los libros impresos ha demostrado que existen tres tipos de de lectores anotadores son: los eruditos y los profesionales, especialmente médicos y juristas, y los profesores y estudiantes. Pues una buena parte de las anotaciones marginales se deben a profesores y alumnos que anotan los libros que poseen durante las mismas clases o durante el estudio (Chartier, 2000, p. 150).

\section{A MODO DE CONCLUSIÓN.}

En el campo de la transmisión de los saberes la imprenta también desencadena el mismo efecto que en el resto de los aspectos produciéndose una continuidad de los usos previos a su aparición y en los primeros años del siglo XVI. No se manifiestan cambios en el contenido de los recursos gráficos y el avance de las nuevas técnicas se produce de forma muy paulatina.

Tampoco hay una evolución apreciable en los textos que se utilizan que son esencialmente los mismos que en el mundo manuscrito. El impresor busca (re)producir aquellas obras que va a vender con la menor dificultad posible y para ello recurrirá a las obras que se copiaron manuscritas y eran más leídas.

La imprenta aporta en estos primeros momentos, como en el resto de los aspectos del conocimiento, algunos productos impresos para el aprendizaje de la lectura, cartillas y juegos de cartas, que conviven con ejemplos manuscritos durante los primeros años del siglo XVI. Este mismo panorama es perceptible en los libros que se utilizan en las enseñanzas posteriormente inmediatas al aprendizaje de la lectura.

En el aprendizaje de los oficios, los nuevos vientos del Renacimiento no comienzan a apreciarse en el mundo del impreso hasta la mitad del siglo XVI, aunque las nuevas formas debieron penetrar en el mundo medieval de mano de cuadernos manuscritos que poseían los artesanos y artistas, generalmente copiados por ellos mismos de ejemplos del natural y, con seguridad, de los grabados y miniaturas que poseían algunos libros venidos desde los principales focos del renacimiento.

Las enseñanzas universitarias, que deberían haber sucumbido a los nuevos avances técnicos de reproducción de documentos y que mejor se verían favorecidos por la normalización y la mejora en la calidad de los textos se constituyen como un reducto, tanto de los contenidos como de las formas. El manuscrito pervive especialmente entre 
los libros de los universitarios, generalmente confeccionados por los propios universitarios mediante la recopilación de obras y de textos heterogéneos que conforman conjuntos cuyo nexo de unión es perfectamente apreciado por el propietario. Pero, a pesar de esta constatación, se aprecia un paulatino ingreso del impreso en el aula, como objeto de estudio y también de comentario y glosa.

No se puede negar, por consiguiente, la importancia que el impreso posee en la transmisión de los saberes en el momento referido, pero no parece que el libro impreso se introdujera de la forma rápida y con la presencia mayoritaria que cabría suponer de un producto tan avanzado. Por tanto, no puede hablarse de un cambio brusco en las formas y, lo que resulta más importante, no parece que el libro impreso (o manuscrito) en las enseñanzas de los saberes esté sirviendo en los primeros momentos del siglo XVI para producir un cambio en los fondos.

\section{NOTAS}

${ }^{1}$ Archivo Histórico de Protocolos Notariales de Zaragoza (desde aquí: A.H.P.N.Z.) Protocolo de Juan Arruego, 1521. En la actualidad desaparecido (Serrano y Sanz, 1914).

2 A.H.P.N.Z. Protocolo de Miguel de Villanueva mayor, 1502, ff. 274 v. / 275. En el que Pedro de San Esteban se reconoce como maestro de enseñar a escribir.

3 Así en la edición electrónica. Debería de ser Sígueme.

4 A.H.P.N.Z. Inventario de bienes de la casa de Gaspar de Terrén e ciertas apocas, Juan de Altarriba, 1504, s. f. (Pedraza, 1993, doc. 200).

5 A.H.P.N.Z. Protocolo de Pedro Serrano, 1506, ff. 88 / 89; o en el protocolo de 1508, f. 57 v., por ejemplo.

6 «Item, un Caton viejo de mano en paper scripto», A.H.P.N.Z. Protocolo de Juan Molés, 1510, ff. 5 / 8 v. Inventario de los bienes del difunto Pedro Quílez, notario.

7 «Doctrinal rasgado de gramática de mano», A.H.P.N.Z. Protocolo de Luis Sora, 1515, ff. 37 / 45 v. Inventario de los bienes de la difunta María Vidal.

${ }^{8}$ Un caso muy especial es el de los ciegos que también firmaban contratos de aprendizaje para recitar y cantar oraciones y posteriormente vender mejor que copias manuscritas, ejemplares impresos. Berenguer Sopeyra, labrador, habitante en Granja de Escarpe (Lérida), cerca de Fraga (Huesca), en el principado de Cataluña, de la Abadía de Escarpe, firma como aprendiz del oficio de tañer guitarra y de "deprender" oraciones, como suelen hacer los ciegos, a su nieto Juanico de Sopeyra, ciego, con García de Serás, músico, vecino de Zaragoza, por doce años. A.H.P.N.Z. Protocolo de Juan de Longares, 1511, ff. 446 / 446 v. de numeración actual.

${ }^{9}$ Como ejemplo, uno de estos casos:

... Yo Gaspar Creer, librero, vezino de Caragoca de mi cierta sciencia otorgo et reconozco a vos Fermin d'Aymar, pelayre, habitant en la ciudat de Caragoca, que por la fianca que me hizisteys et entrasteys en la carta quando vuestro nieto, Pedro de Gurrea, fijo de Miguel Gurrea, habitant las oras en Caragoca, se firmo con mi al officio mio de librero por tiempo que tres anyos segunt consta por carta fecha en Caragoça a treze de setiembre anyo mil quatrozientos noventa y seys, testificada por el discreto Johan de Aguas menor, notario publico de Caragoça, me haveys dado vinticinquo sueldos jaqueses y las expensas del proceso y esto por las expensas et danyos que he recibido por quanto el moço se me fue de mi servicio.

A.H.P.N.Z., Formulario de Pedro Lalueza, 1495-1500, ff. 8v. $/ 9$.

10 Juan de Labasuy, natural de Graus (Huesca), habitante en Zaragoza, firma como aprendiz de librero con Luis Trincher, librero, vecino de Zaragoza, durante tres años. Luis Trincher se obliga, además, a enseñarle a leer y a escribir. A.H.P.N.Z., Protocolo de Luis Sora, 1522 , ff. 439 v. / 440.

${ }^{11}$ Pedraza, 1993, doc. 651. En este proceso de la inquisición se puede observar como se utiliza un ejemplar manuscrito de un grimorio para hacer caer en la trampa al comerciante Pedro Bernardi para robarle y hacerse con una copia que habría realizado él mismo.

12 A.H.P.N.Z. Protocolo de Pedro Lalueza, 1511, ff. 515 v. / 516. El 19 de octubre de 1511 Juan Rodrigo, habitante en Zaragoza, maestro en artes y bachiller en teología, reconoce haber recibido «una biblia con su comento et ocho volumenes otros de libros» de los albaceas del testamento de Juan Fernández Arrojo, presbítero, difunto, por manos de Juan Crespo, albacea del mencionado testamento, los cuales tenía empeñados como garantía de 4'5 florines que le había prestado el mencionado Juan Fernández Arrojo.

13 A.H.P.N.Z. Juan de Longares, Liber Llegatorum, 1498, 2 papeles sueltos.

14 A.H.P.N.Z. Protocolo de Pedro Serrano, 1506, ff. 120 / 122 v.

15 A.H.P.N.Z. Protocolo de Jimeno Gil, 1502, ff. 178 / 181

16 A.H.P.N.Z. Protocolo de Alfonso Francés, 1503, ff. 89 / 91 v.

17 A.H.P.N.Z. Protocolo de Pedro Lalueza, 1511, ff. 207 / 222 v.

18 A.H.P.N.Z. Protocolo de Juan de Altarriba, 1506, suelto s. f.

19 A.H.P.N.Z. Protocolo de Antón Tomás menor, 1501, s. f.

${ }^{20}$ A.H.P.N.Z. Protocolo de Pedro Martínez de Insausti, 1514, ff. 124 v. / 125.

21 A.H.P.N.Z. Protocolo de Luis Sora, 1517, ff. 126 v. / 127.

\section{BIBLIOGRAFÍA.}

ÁLVAREZ MÁRQUEZ, M.C. La enseñanza de las primeras letras y el aprendizaje de las artes del libro en el siglo XVI en Sevilla. Historia, instituciones, documentos, 1995, n 22, p. 39-86.

BERGER, P. Libro y lectura en la Valencia del Renacimiento. Valencia: Alfons el Magnànim, 1987, 2 vols.

BOUZA ÁlVAREZ, F.J. Del escribano a la biblioteca: la civilización escrita europea en la Alta Edad Moderna (siglos XV-XVI). Madrid: Síntesis, 1992. 
CHARTIER, R. Las revoluciones de la cultura escrita: diálogo e intervenciones. Barcelona: Gedisa, 2000.

EISENSTEIN, E. The printing press as an agent of change: communications and cultural transformations in early modern Europe. New York: Cambridge University Press, 1979, 2 vols. Traducción al castellano en: México: Fondo de Cultura Económica.

EISENSTEIN, E. La revolución de la imprenta en la Edad Moderna. Madrid: Akal, 1994.

GRAFTON, A. The culture of correction in Renaissance Europe. London: The British Library, 2011.

GRAFTON, A. La page, de l'Antiquité à l'ère du numérique: histoire, usages, esthétiques, Paris: Hazan, 2012.

INFANTES, V. De las primeras letras: cartillas españolas para enseñar a leer de los siglos XV y XVI. Salamanca: Universidad de Salamanca, 1998.

INFANTES, V. La educación impresa. Cuadernos de Historia Moderna. Anejos, Anejo III, Monografías (Ingenios para el mundo: sociedad, saber y educación en la Edad Moderna, Teresa Nava Rodríguez [coord.]), 2004, p. 227251.

MCLUHAN, M. La galaxia Gutenberg: génesis del homo typographicus. Madrid: Aguilar, 1969.

PEDRAZA GRACIA, M.J. Documentos para el estudio de la historia del libro en Zaragoza entre 1501 y 1521. Zaragoza: Centro de Documentación Bibliográfica, 1993.

MARTÍNEZ PEREIRA, A. Manuales de escritura de los Siglos de Oro: repertorio crítico y analitico de obras manuscritas e impresas. Mérida: Editora Regional de Extremadura, 2006.

RUIZ GARCÍA, E. La balanza y la corona: la simbólica del poder y los impresos jurídicos castellanos (1480-1520). Madrid: Ollero y Ramos, 2011.

SÁNCHEZ HERRERO, J. y PÉREZ GONZÁLEZ, S.M. Aprender a leer y escribir, libros y libreros en la Sevilla del último cuarto del siglo XV. Edad Media: revista de Historia, 1998, n 1, p. 47-90.

SERRANO Y MORALES, J.E. Reseña histórica en forma de diccionario de las imprentas que han existido en Valencia desde la introducción del arte tipográfico en España hasta el año 1868 con noticias bio-bibliográficas de los principales impresores. Valencia: F. Doménech, 1988-1989.

SERRANO Y SANZ, M. Testamento de Gonzalo García de Santa María. Boletín de la Real Academia Española, 1914, año 1, t. 1, n $^{\circ} 5$, p. 470-478.

SOCIAS BATET, I. Cataleg del Fons Abadal de la Biblioteca de Catalunya. Barcelona: Biblioteca de Catalunya, 2006.

SOCIAS BATET, I. Les Beceroles tabel-làries de la Biblioteca de Catalunya. Barcelona: Biblioteca de Catalunya, 2006.

VIÑAO FRAGO, A. Alfabetización y primeras letras. En Escribir y leer en el siglo de Cervantes. Barcelona: Gedisa, 1999, p. 39-84.

VIVES, J.L. Diálogos. Buenos Aires: Espasa Calpe, 1999. Ed. digital [en línea]. Disponible en: $<$ http://www.cervantesvirtual.com/servlet/SirveObras/35704175214571495222202/p0000001.htm>. [Consulta: 8 de abril de 2013].

YCIAR, J.de. Recopilación subtilissima intitulad orthographia practica. Zaragoza: Pedro Bernuz, 1548. 